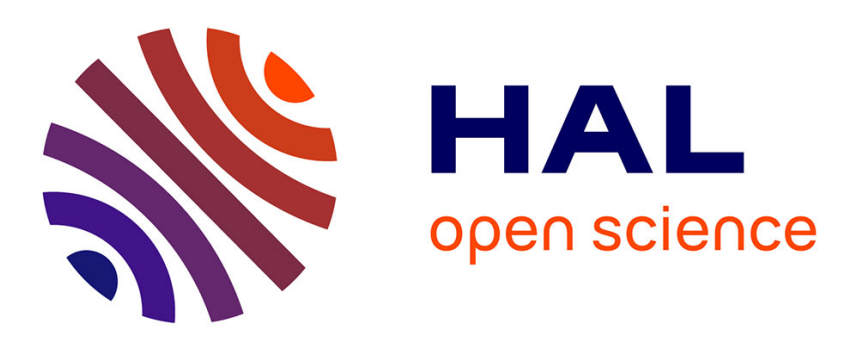

\title{
Une autre géographie du développement rural: une approche par les revenus
}

\author{
Magali Talandier
}

\section{To cite this version:}

Magali Talandier. Une autre géographie du développement rural: une approche par les revenus. Géocarrefour - Revue de géographie de Lyon, 2008, 83 (4), pp.259-267. halshs-00851932

\section{HAL Id: halshs-00851932 \\ https://shs.hal.science/halshs-00851932}

Submitted on 19 Aug 2013

HAL is a multi-disciplinary open access archive for the deposit and dissemination of scientific research documents, whether they are published or not. The documents may come from teaching and research institutions in France or abroad, or from public or private research centers.
L'archive ouverte pluridisciplinaire $\mathbf{H A L}$, est destinée au dépôt et à la diffusion de documents scientifiques de niveau recherche, publiés ou non, émanant des établissements d'enseignement et de recherche français ou étrangers, des laboratoires publics ou privés. 


\section{Une autre géographie du développement rural : une approche par les revenus.}

Magali Talandier

Docteur en Urbanisme, politiques urbaines et aménagement

Institut d'Urbanisme de Paris, Université Paris 12 - Val de Marne

magali@talandier.com

\section{$\underline{\text { Résumé }}$}

Les territoires ruraux éloignés des métropoles semblaient condamnés au déclin démographique et économique. Or, ils attirent de nouveaux résidents, enregistrent une progression rapide des emplois et du revenu. La capacité productive des territoires ne suffit plus à expliquer ces nouvelles tendances, la périurbanisation non plus. Les revenus de transferts, les salaires des agents de la fonction publique, les retraites, les dépenses des touristes... sont autant de revenus qui induisent, également, des dynamiques de développement local et dessinent une autre géographie du développement rural.

\section{$\underline{\text { Mots clés }}$}

Développement local, Économie résidentielle, Développement rural, Théorie de la base

\section{$\underline{\text { Abstract }}$}

The rural regions further away from large towns could decline demographically and economically. But the remote rural areas are attracting more and more residents. This demographic recovery accompanies a significant progression of employment and income, and this development of non-metropolitan areas questions both the theory and the experts. The periurbanisation and the productive capacity of these regions now seem insufficient to explain these new trends. State transfer revenue, civil servant salaries, retirement pensions, tourist spending... are contributing factors to local development and are taking shape a new geography of rural development.

$\underline{\text { Key Words }}$

Local development, Residential economy, Rural development, Economic base theory

\section{Introduction}

Après un siècle d'exode rural et une perte démographique estimée à $40 \%$ de la population ${ }^{1}$, la désertification des campagnes semblait inéluctable. Pourtant, dès les années 1970, les soldes migratoires redeviennent positifs dans de nombreuses communes rurales périurbaines ou situées dans des départements très touristiques. Ces migrations se confirment et s'accélèrent à chaque recensement jusqu'à gagner le rural isolé ${ }^{2}$ en 1999.

Ces mutations démographiques s'accompagnent de changements économiques, sociaux, et culturels qui obligent à repenser le monde rural (Kayser, 1990; Bontron, 1993 ; Renard,

\footnotetext{
${ }^{1}$ Ces chiffres reflètent le solde entre l'exode rural et le retour rural et non, de façon stricte, les mouvements des campagnes vers les villes, cf. Merlin (1971).

${ }^{2}$ Le rural isolé désigne dans le ZAUER (zonage en aires urbaines et emplois de l'espace rural - INSEE, INRA, 1998), l'ensemble des communes de l'espace rural qui ne sont ni pôle rural, ni couronne d'un pôle rural, ni sous faible influence urbaine (ainsi ces communes ne sont pas des pôles d'emplois (soit moins de 2000 emplois) et moins de $20 \%$ de leurs actifs travaillent dans un pôle urbain, une aire urbaine, ou un pôle rural).
} 
2000 ; Perrier-Cornet, 2002 ; Ceramac, 2007), mais qui obligent également à repenser les modèles d'analyse du développement local. Les avantages comparatifs territoriaux se modifient, et ce qui condamnait, hier et sans appel, les territoires au déclin n'est plus d'évidence aujourd'hui. La géographie de la croissance est de moins en moins représentative de la géographie du développement. La concentration dans les métropoles des activités stratégiques, celles qui créent le plus de valeur ajoutée, s'est accompagnée au cours des vingt dernières années d'une diminution des disparités territoriales en termes de revenu des populations (Davezies, 2008). C'est dans ce contexte de déconnexion entre les facteurs de croissance et ceux du développement, que les territoires ruraux, même isolés, attirent de nouvelles populations, créent des emplois, enregistrent de fortes hausses du revenu.

Depuis quelques années, le terme d'économie résidentielle, ou encore les notions de fonction résidentielle et récréative, sont mobilisés pour évoquer ce renouveau rural. Mais, qu'entendent les chercheurs et les experts lorsqu'ils se réfèrent à la sphère résidentielle des économies locales ? La théorie de la base ${ }^{3}$, formulée en termes de flux de revenus, constitue l'un des cadres théoriques pertinents pour éclaircir cette question.

Enfin, au-delà de la composante résidentielle des économies locales, cette approche par les revenus permet de dresser une géographie singulière du développement rural actuel et fait émerger des interrogations sur la durabilité de certains processus.

\section{La revitalisation rurale interroge les cadres analytiques habituellement mobilisés en développement local}

Les modèles de la Nouvelle Economie Géographique (Krugman, 1991 ; Combes, Mayer, Thisse, 2006 pour une revue récente de la littérature) n'ont certes pas pour objectif d'offrir un cadre analytique aux questions de développement local, et rural qui plus est. Néanmoins, ces

\footnotetext{
${ }^{3}$ La théorie de la base est probablement l'un des cadres conceptuels les plus utilisés en analyse régionale au cours des années 1950/1980.

Formulé initialement par Cantillon (1755) dans son Essai sur la nature du commerce en général, puis par Sombart (1916) dans son Der Moderne Kapitalismus, le principe de cette théorie repose sur une idée simple selon laquelle les économies locales peuvent se décomposer en deux secteurs : i) un secteur qui permet de capter les revenus extérieurs, appelé secteur basique et qui constitue le moteur de l'économie locale et ii) un secteur induit par ces revenus captés qui répond à la demande locale de biens et de services, appelé secteur domestique.

A partir des années 1950, des auteurs comme Hoyt, North ou encore Tiebout reprennent ce principe et l'appliquent non pas sur des flux monétaires ou revenus mais sur les emplois. Ils désignent par le calcul de coefficients de localisation, les activités exportatrices inductrices de développement et les activités ou emplois induits. Face à la déconnexion croissante entre les résultats issus de ces analyses et les faits, cette théorie sera vivement critiquée et abandonnée au cours des années 1980.

En 1990, on assiste à la réémergence de cette théorie grâce notamment en France à Loiez Laurent (1995) ou encore à Laurent Davezies. Ces auteurs montrent qu'en effet la théorie de la base limitée à son approche en termes d'activités exportatrices (industries, services aux entreprises etc.) est trop limitative et qu'il faut aujourd'hui considérer l'ensemble des mécanismes de captation de revenu et non pas uniquement les exportations de biens et services. Parmi ces autres mécanismes dont bénéficient les territoires se trouvent les revenus de la redistribution publique et sociale, les retraites, le tourisme, les revenus des navetteurs, etc.
}

Les lecteurs intéressés retrouveront dans Gonnard (2001) le récit complet du parcours tourmenté de la théorie de la base dans l'histoire de la pensée économique jusqu'à la fin du $\mathrm{XX}^{\mathrm{e}}$ siècle. 
modèles alimentent depuis plus de quinze ans les réflexions menées dans les pays industriels en la matière. Concernant les espaces ruraux, ces modèles mettent en avant les effets de dispersion que peuvent générer les agglomérations (congestion urbaine, pression foncière, pollution, etc.) Ces coûts et nuisances induisent la périurbanisation des ménages et des emplois dont «bénéficient » les communes rurales périphériques (Gaigné, 2000, Gaigné et alii, 2003). En revanche, ces modèles peinent à expliquer le développement des espaces ruraux non périurbains.

Contrairement à ces modèles, les approches du développement endogène et les travaux sur les systèmes de production localisés (SPL) portent souvent sur des territoires ruraux (Beccatini, 1992 ; Pecqueur, 1989, Courlet, Pecqueur, 1992). Ce développement par et pour les acteurs s'appuie sur la valorisation de ressources spécifiques, territorialement ancrées, qu'il s'agisse d'un savoir-faire particulier, d'une histoire collective, de la qualité d'un terroir. La mobilisation - ou activation (François et alii, 2006) - de ces ressources territoriales, imparfaitement délocalisables, offriraient aux espaces ruraux une alternative de développement face à la métropolisation et à la mondialisation des échanges (Coissard et Pecqueur, 2007). Ainsi, il n'y aurait pas de fatalité de sous-développement pour les territoires non métropolitains. Pour autant, peut-on assurer que ces systèmes de production localisés suffisent à expliquer la revitalisation rurale ? Rien n'est moins sûr ${ }^{4}$. Comme le notent Colletis et Rychen (2004), "l'ancrage territorial des activités industrielles correspond à une valorisation localisée de l'activité d'une entreprise. Toutefois, ce mode de valorisation du territoire par l'entreprise ne signifie pas que l'entreprise contribue forcément au développement du territoire. » (p.215 in Pecqueur, Zimmermann, dir., 2004).

Finalement, à la lecture de ces travaux, un constat s'impose : sans la proximité d'un pôle urbain dynamique ou l'existence d'un appareil productif spécifique et en «bonne santé », les espaces ruraux seraient condamnés au déclin démographique, économique, social.

Or, entre 1993 et 2005 l'emploi salarié privé (emploi Unedic) a progressé plus rapidement dans le rural isolé $(20,5 \%)$ qu'en moyenne en France métropolitaine (19,3\%). Les plus fortes progressions sont enregistrées dans les communes périurbaines $(38,3 \%)$, puis dans le rural isolé $(20,5 \%)$; viennent ensuite le rural sous faible influence urbaine (18,3\%), les pôles urbains $(17,7 \%)$, les pôles ruraux $(16,7 \%)$, les communes multipolarisées $(15,7 \%)$. Les évolutions ne semblent donc pas dépendre de la plus ou moins grande urbanité de la commune. Ces évolutions seraient-elles soutenues par la qualité et les spécificités du tissu productif local ? Difficile d'amender cette hypothèse, puisque les emplois Unedic productifs ${ }^{5}$ diminuent de $-1,1 \%^{6}$ dans le rural isolé entre 1993 et 2005 (hors agriculteurs-exploitants

\footnotetext{
${ }^{4}$ Callois (2004) se demande si les signes de qualité sur les produits alimentaires peuvent engendrer, par effets induits, une croissance économique, voire l'arrivée de nouvelles populations. Ses conclusions sont plutôt mitigées. La rente créée bénéficie à une minorité d'agriculteurs, mais l'impact social peut se révéler positif en termes de cohésion et d'identité locale. Les préférences des consommateurs vont également influer sur le volume demandé en biens manufacturés typiquement ruraux. Quant à l'impact sur les migrations, il dépendrait du dynamisme du marché de l'emploi local et des niveaux de salaires. Notons que l'image véhiculée par ces produits et sa probable attractivité sur les inactifs n'est pas réellement testée.

5 Emplois dans les secteurs qui se localisent pour produire et exporter (industrie, recherche, vente par correspondance, audiovisuel, etc.)

${ }^{6}$ Ce type d'emplois diminue de $10,2 \%$ en moyenne en France sur la même période. À propos de ce relatif maintien de l'industrie rurale voir Aubert et Blanc (2002).
} 
puisque l'on ne considère que les emplois salariés). Ce sont les secteurs tournés vers la demande de biens et services consommés localement (commerces de détail, services aux personnes), les secteurs directement liés au tourisme (hôtels, restaurants), les activités de BTP qui dynamisent le marché de l'emploi dans le rural isolé. Dynamisme de l'emploi salarié privé (mais également de l'emploi non salarié ${ }^{7}$ ) dans le rural isolé, auquel s'additionnent les plus fortes progressions du revenu ${ }^{8}$ par habitant sur les vingt dernières années.

Emploi, revenu : qu'en est-il de la population et de l'attractivité migratoire ?

L'un des enseignements majeurs du recensement de 1999 a été de révéler que les soldes migratoires étaient à présent positifs dans le rural isolé. Entre 1990 et 1999, l'analyse de ces mobilités résidentielles permet d'identifier que les flux les plus importants se font des pôles urbains vers le périurbain, puis vers le rural sous faible influence urbaine, puis vers le rural isolé (Sencébé, Le Picier, 2005). Implicitement, on suppose que ces flux des pôles urbains (ou du périurbain) vers les espaces ruraux concernent des communes géographiquement proches et témoignent de l'étalement urbain. En introduisant la distance parcourue par les émigrants des aires urbaines, on constate que pour plus d'une installation sur deux dans le périurbain, la distance parcourue est, en effet, inférieure à trente kilomètres. On quitte le centre pour la périphérie. Il en va tout autrement dans les espaces ruraux. Dans le rural isolé, près de $45 \%$ des urbains nouvellement installés ont parcouru plus de deux cent kilomètres. Continuer à parler d'étalement urbain n'a guère de sens.

La géographie des mobilités (carte 1) montre que certaines communes sont à la fois peu attractives auprès des urbains «proches » et des urbains «éloignés» $(200 \mathrm{~km})$. Elles sont situées dans le Nord-Est, à l'est de la Bretagne, en Saône-et-Loire, dans une partie des Pyrénées-Atlantiques.

Le regain démographique des espaces ruraux du Grand Bassin parisien, du pourtour de l'aire urbaine de Lyon, de Nantes, de Rennes, de Lille, de Metz, de Nancy, de Strasbourg, de Mulhouse s'explique avant tout par le mécanisme d'étalement urbain.

De nombreuses communes sont très attractives auprès des migrants de longue distance mais peu auprès des urbains ou périurbains proches. La majorité des urbains venus s'y installer résidaient donc à plus de $200 \mathrm{~km}$ en 1990. Plus précisément, dans ces communes situées plutôt dans le Centre, le Sud-Ouest, en Bretagne, sur le pourtour méditerranéen ou encore dans les Alpes, 37\% de ces urbains nouvellement installés viennent de l'aire urbaine de Paris. Il paraît donc difficile de comprendre l'attractivité migratoire de ces espaces à la seule lumière de l'étalement urbain.

Enfin, les communes à la fois très attractives auprès des urbains proches et des urbains venus de plus loin sont situées dans les départements languedociens, au pourtour d'aires urbaines dynamiques comme Toulouse, Rennes, Bordeaux. La concentration de ce type de communes

\footnotetext{
${ }^{7}$ Le nombre de travailleurs indépendants en profession libérale progresse de 17,8\% entre 1999 et 2005 dans le rural isolé, contre 13,3\% en moyenne en France. Le nombre de travailleurs indépendants déclarant des bénéfices industriels et commerciaux (artisans, commerçants, agents commerciaux) diminue moins rapidement dans le rural isolé $(-2,3 \%)$ qu'en France métropolitaine $(-5,1 \%)$ - source Sirene, calculs de l'auteur.

${ }^{8}$ Revenu déclaré aux impôts, source DGI de 1984 à 2004. Calcul des revenus par habitant effectué par l'auteur, cf. Talandier (2007).
} 
dans le Sud-Est est notable et illustre l'attractivité conjointe auprès de migrants franciliens ou étrangers, mais également le reflux des capitales régionales vers les arrière-pays.

Un travail similaire ne peut pas être effectué sur des données plus récentes pour l'instant en raison du changement de méthode du recensement de la population, mais on peut penser que cette attractivité migratoire de longue distance se poursuit dans le rural. En effet, sur la période 2004-2006, la part des migrants régionaux ${ }^{9}$ dans la population est plus importante dans les espaces ruraux que dans les espaces urbains en général, plus importante dans le rural isolé et le rural sous faible influence urbaine en particulier. La géographie de ces migrations interrégionales récentes confirme la forte attractivité des espaces ruraux du Sud et de l'Ouest.

\footnotetext{
${ }^{9}$ Personnes résidant dans la commune lors de l'enquête du recensement INSEE (depuis 2004) et qui résidait dans une autre région au cours des cinq années précédentes.
} 
Carte 1 - Typologie des communes hors aires urbaines selon la part des immigrants dans la population 1999 venant d'une aire urbaine située à moins de 200 kilomètres ou à plus de 200 kilomètres.

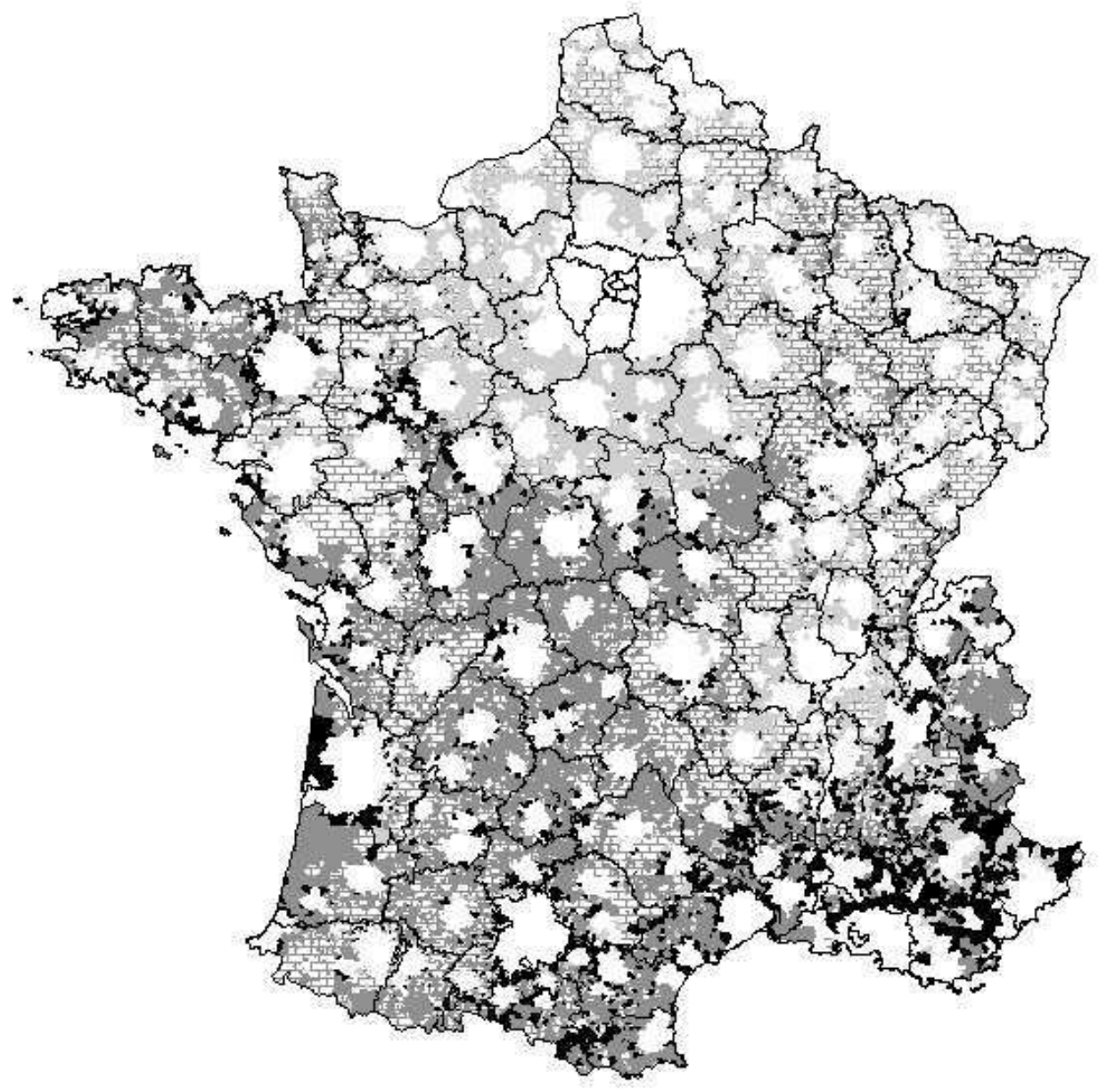

Part des immigrants venant d'une aire urbaine située à moins de $200 \mathrm{~km}$ ("immigrants périurbains") et part des immigrants venant d'une aire urbaine située à plus de $200 \mathrm{~km}$ ("immigrants de longue distance") dans la population 1999, par comparaison à la moyenne française.

도 Moins attractives que la moyenne française auprès des immigrants périurbains et de longue distance.

Plus attractives que la moyenne française auprès des immigrants périurbains mais moins attractives auprès des immigrants de longue distance.

Moins attractives que la moyenne française auprès des immigrants périurbains mais plus attractives auprès des immigrants de longue distance.

Plus attractives que la moyenne française auprès des immigrants périurbains et de longue distance.

Source : INSEE, calculs de l'auteur - Talandier (2007), p.128.

En blanc : les aires urbaines. 
Forte attractivité migratoire au-delà du seul phénomène de desserrement urbain, essor des emplois de commerces, de services, succès des activités touristiques : une troisième voie se dessine pour comprendre la revitalisation rurale en cours, celle de l'économie résidentielle. Mais que recouvre exactement cette notion?

\section{La théorie de la base comme cadre analytique ou une approche par les revenus}

\section{La théorie de la base pour définir la sphère résidentielle des économies locales}

L'expression « économie résidentielle » est devenue, en quelques années, un des maîtres mots du développement local. Sa définition reste néanmoins soumise à discussion.

L'INSEE, par exemple, s'est emparé de ce thème pour désigner les emplois domestiques, c'est-à-dire ceux tournés vers la demande locale: commerces de détail, services aux personnes, etc. On peut souligner que la méthodologie qui permettrait de qualifier la nature domestique ou non d'une activité n'est pas discutée, alors qu'elle pose de lourdes et complexes questions conceptuelles.

Davezies (2003) introduit cette notion pour distinguer les flux de revenus que capte un territoire grâce à ses atouts résidentiels. L'allongement de la durée de vie, la hausse des revenus, les progrès technologiques (TGV, téléphone portable, etc.) ont eu raison de la logique «un individu - un territoire ». La «multi-appartenance» territoriale explique qu'aujourd'hui, la production et la consommation peuvent être déterminées par des «lois » géographiques et économiques différentes. L'économie résidentielle s'attache à ce qui motive les choix résidentiels et récréatifs des individus; l'économie productive s'inscrit dans une lecture plus classique de localisation des firmes et des travailleurs (proximité des facteurs de production, économies d'agglomération). Les deux concordent parfois, divergent souvent.

Le concept de la théorie de la base (Sombart, 1916) revisité par Davezies (2003) est l'une des rares approches qui permettent de considérer conjointement ces différents aspects du développement local (moteurs productifs et non productifs ; impact de la consommation sur le développement). Le secteur basique désigne l'ensemble des revenus qu'un territoire capte de l'extérieur. Le secteur domestique répond à la demande locale de biens et services. Véritable moteur de l'économie locale, le secteur basique induit, par un jeu de multiplicateur, l'essor du secteur domestique et assure le dynamisme économique et démographique des territoires. Davezies (2003) propose de distinguer quatre types de revenus basiques ou bases :

- la base productive privée est constituée des revenus (salaires ou bénéfices) perçus par les habitants des territoires, grâce à l'implantation d'entreprises qui produisent et exportent en dehors de la zone étudiée ;

- la base résidentielle regroupe les revenus captés par les territoires grâce à leurs « atouts » résidentiels. Il s'agit plus précisément des salaires « rapatriés » au lieu de domicile par les migrants alternants ; des retraites ; des dépenses des touristes ; 
- la base publique est constituée des traitements des trois fonctions publiques : fonction publique d'État, hospitalière et territoriale ${ }^{10}$;

- la base sanitaire et sociale comprend l'ensemble des revenus de transferts : minima sociaux, allocations chômage, allocations familiales, allocations de logement, indemnités journalières, remboursements de soins de santé ${ }^{11}$.

Deux types d'enjeux de développement local coexistent et cohabitent plus ou moins bien : un enjeu de production (le développement dépend de la qualité du système productif local, de la capacité à attirer les entreprises et à favoriser leur essor) et un enjeu de population (le développement dépend de la population importatrice de revenus qui alimentent l'économie locale, indépendamment de sa capacité productive).

Les revenus basiques, ou importés, ne constituent qu'une potentialité de développement pour le territoire et une des questions-clés du développeur local est de transformer ces revenus en demande. Schématiquement, tout se passe selon un mécanisme néo-classique en amont (capter des revenus) et keynésien en aval (transformer ces revenus en consommation locale). Maximiser la propension à consommer localement permet d'entretenir le marché de l'emploi domestique qui peut apporter une réponse au chômage des jeunes peu ou pas qualifiés ${ }^{12}$. Si ces emplois sont occupés par des locaux, ils peuvent induire une baisse de la pauvreté, du chômage, attirer des actifs. La situation de la population locale a donc tendance à s'améliorer, la demande va s'en trouver dynamisée : le processus de développement se poursuit.

\section{Estimations des revenus basiques des bassins de vie français}

Le calcul ${ }^{13}$ des revenus basiques a été réalisé à l'échelle des bassins de vie de l'INSEE $^{14}$ en France métropolitaine, et détaillé selon la nature de la commune qui polarise le bassin de vie. Les résultats sont repris dans le tableau 1.

Selon ces estimations, la base résidentielle représente en 2005 entre 40,7\% (pôle urbains de plus de 30000 habitants) et plus de $70 \%$ (communes périurbaines) des revenus basiques. Ainsi, même dans les pôles urbains de plus de 30000 habitants, la première source de revenus n'est pas issue de son système productif, mais de ses retraités ! Cette approche révèle le poids des revenus résidentiels dans l'analyse des dynamiques territoriales, puisque près de la moitié des revenus basiques des bassins de vie provient de la base résidentielle. Viennent ensuite, avec plus de $20 \%$, la base sociale et sanitaire ( $12 \%$ pour le social et $10 \%$ pour la santé), la

\footnotetext{
${ }^{10}$ Les traitements de la fonction publique territoriale sont financés en grande partie par les dotations de l'État. En concertation avec des experts de la question, la part basique de ce type de salaires est estimée à $70 \%$; 30\% seraient donc financés directement par les collectivités territoriales.

${ }^{11}$ Ce sont les remboursements de soins de santé qui sont considérés comme basiques et non les bénéfices des professions médicales. Cela revient à considérer que ce sont les malades qui sont à l'origine de ces revenus et que ces remboursements induisent directement l'emploi domestique du secteur médical privé.

${ }^{12}$ Les emplois domestiques offrent une large palette de qualification, du médecin, pharmacien, notaire à l'employé de commerce, l'assistante maternelle ou la femme de ménage.

${ }^{13}$ Une méthodologie détaillée de ces calculs est disponible dans Talandier (2007, vol.II, pp.54-71).

${ }^{14}$ INSEE et autres sources, 2003.
} 
base productive $(19,7 \%)$ et la base publique $(9,1 \%)$. Ainsi, comprendre le développement rural (et plus généralement local) à la seule lecture du système productif parait insuffisant.

Dans le rural isolé, avec plus d'un tiers des revenus basiques, le tourisme apparaît comme le premier moteur économique. Les pôles ruraux apparaissent plus productifs que le rural sous faible influence urbaine, lui-même plus productif que le rural isolé. La base publique est relativement faible dans ces espaces (entre 4 et 5\%). Enfin, le poids des revenus sociaux se situe autour de $11 \%$, celui des remboursements de soins de santé entre 7 et $8 \%$, proportion inférieure à la moyenne des bassins de vie français.

Tableau 1 - Répartition des revenus basiques par bassin de vie selon le type de pôle du bassin, en \% (en 2004/2005)

\begin{tabular}{|c|c|c|c|c|c|c|c|c|}
\hline & $\begin{array}{l}\text { Pôle urbain } \\
>30.000 \text { hab }\end{array}$ & $\begin{array}{l}\text { Pôle urbain } \\
<30.000 \text { hab }\end{array}$ & $\begin{array}{l}\text { Commune } \\
\text { périurbaine }\end{array}$ & $\begin{array}{c}\text { Commune } \\
\text { multipolarisée }\end{array}$ & $\begin{array}{l}\text { Pôle } \\
\text { rural }\end{array}$ & $\begin{array}{l}\text { Rural } \\
\text { isolé }\end{array}$ & $\begin{array}{c}\text { Rural sous } \\
\text { faible } \\
\text { influence } \\
\text { urbaine }\end{array}$ & \begin{tabular}{|c|} 
Ensemble \\
des \\
bassins de \\
vie
\end{tabular} \\
\hline Total base productive & 23,8 & 15,4 & 9,6 & 10,7 & 14,1 & 10,7 & 12,1 & 19,7 \\
\hline \begin{tabular}{|l|l|} 
Total base résidentielle \\
\end{tabular} & 40,7 & 55,6 & 70,3 & 67,3 & 61,3 & 67,5 & 65,0 & 49,0 \\
\hline Salaires des migrants-alternants & 7,2 & 14,8 & 41,3 & 35,2 & 16,2 & 11,0 & 23,0 & 12,9 \\
\hline Retraites & 23,3 & 24,2 & 19,0 & 21,3 & 23,9 & 23,7 & 24,7 & 23,2 \\
\hline Dépenses touristiques totales & 10,1 & 16,5 & 10,0 & 10,9 & 21,2 & 32,7 & 17,3 & 13,0 \\
\hline \begin{tabular}{|l|} 
Total base publique \\
\end{tabular} & 11,5 & 7,4 & 3,4 & 3,6 & 5,1 & 4,0 & 4,0 & 9,1 \\
\hline Base publique territoriale & 3,2 & 2,1 & 1,3 & 1,4 & 1,7 & 1,6 & 1,7 & 2,6 \\
\hline Base publique d'Etat & 5,7 & 3,4 & 1,7 & 1,8 & 2,4 & 1,9 & 2,0 & 4,4 \\
\hline Base publique hospitalière & 2,7 & 2,0 & 0,3 & 0,4 & 1,0 & 0,5 & 0,3 & 2,0 \\
\hline \begin{tabular}{|l|} 
Total base sociale et sanitaire \\
\end{tabular} & 24,0 & 21,6 & 16,8 & 18,3 & 19,5 & 17,8 & 18,9 & 22,2 \\
\hline social & 12,4 & 11,6 & 9,9 & 10,9 & 11,3 & 10,5 & 11,7 & 11,9 \\
\hline Allocations ASSEDIC & 3,8 & 3,1 & 3,1 & 3,2 & 2,9 & 2,3 & 2,9 & 3,5 \\
\hline Indemnités Journ. maladie / maternité & 1,2 & 1,1 & 1,1 & 1,1 & 1,0 & 0,8 & 1,0 & 1,2 \\
\hline RMI & 0,7 & 0,4 & 0,2 & 0,3 & 0,4 & 0,4 & 0,4 & 0,6 \\
\hline Prestations fam. et alloc. logts & 4,6 & 4,9 & 3,9 & 4,6 & 5,0 & 4,8 & 5,4 & 4,7 \\
\hline Minimum vieillesse et invalidité & 0,2 & 0,2 & 0,1 & 0,1 & 0,2 & 0,3 & 0,2 & 0,2 \\
\hline $\begin{array}{r}\text { Autres (allocations aux adultes } \\
\text { handicap., aides aux personnes } \\
\text { âgées, aides à l'enfance...) }\end{array}$ & 1,8 & 1,9 & 1,4 & 1,6 & 1,9 & 1,9 & 1,9 & 1,8 \\
\hline santé & 11,6 & 10,0 & 6,9 & 7,4 & 8,2 & 7,3 & 7,2 & 10,4 \\
\hline Pratiques médicales & 3,7 & 2,9 & 1,6 & 1,6 & 1,9 & 1,6 & 1,5 & 3,0 \\
\hline Pharmarcie & 3,1 & 3,1 & 2,4 & 2,7 & 2,8 & 2,8 & 2,8 & 3,0 \\
\hline $\begin{array}{l}\text { Autres (actes auxilliaires, biologie } \\
\text { prélèvements, biens médicaux...) }\end{array}$ & 4,9 & 4,6 & 3,8 & 4,3 & 4,2 & 4,2 & 4,3 & 4,7 \\
\hline \begin{tabular}{|l|l|} 
Total revenus basiques \\
\end{tabular} & 100,0 & 100,0 & 100,0 & 100,0 & 100,0 & 100,0 & 100,0 & 100,0 \\
\hline
\end{tabular}

Source : calculs de l'auteur d'après DGI, INSEE, CAF, SAE, DREES, MSA, Ecosanté, Ministère du Tourisme.

Ces moyennes par bassin de vie masquent de fortes disparités entre les bassins, disparités qui dessinent une géographie des moteurs du développement rural. 


\section{Coexistence de modes de développement différenciés dans les espaces ruraux, dynamiques et incidences des processus en cours.}

\section{Une géographie des moteurs du développement rural}

Une typologie ${ }^{15}$ des 1050 bassins de vie ruraux ${ }^{16}$ a été construite à partir des revenus basiques en France métropolitaine. L'analyse en composante principale et la classification conduisent à identifier six types de territoires. Tous ces bassins restent dominés par les revenus résidentiels, mais certains apparaissent plus productifs, ou plus touristiques que les autres. Les qualificatifs utilisés pour désigner ces six types reprennent les noms des revenus basiques qui les distinguent (cartes 2, 3, 4 où pour plus de lisibilité les six types ont été cartographiés par paires).

Cartes 2, 3 et 4 - Typologie des bassins ruraux établie selon les revenus basiques en 2004/2005
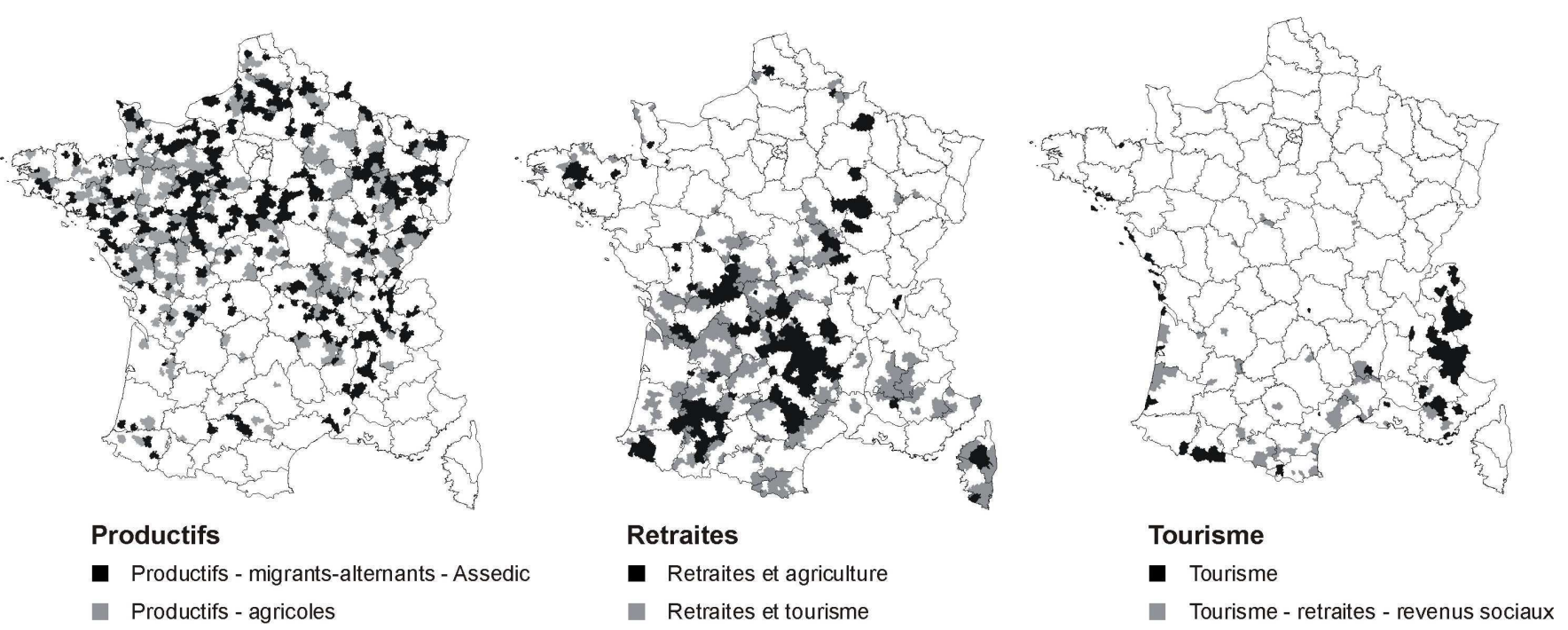

M. Talandier, 2008.

Dans la moitié nord du pays, les bassins ruraux du type «productifs - migrants alternants et Assedic » se situent plutôt à proximité d'une aire urbaine et sur le pourtour francilien. Le type « productif et agricole » domine à l'Ouest ainsi que dans certains départements agricoles ou viticoles des régions de l'Est : Champagne-Ardenne, Bourgogne et Franche-Comté. Toujours dans cette moitié nord, certains bassins ruraux sont moins attractifs auprès de jeunes actifs ou peut-être plus attractifs auprès de retraités - puisque leur base est dominée par les retraites. Ces bassins de retraités sont également plus touristiques que la moyenne près du littoral des

\footnotetext{
${ }^{15}$ La typologie a été réalisée en deux temps. Une analyse en composante principale a d'abord permis de sélectionner les variables (ou bases) les plus discriminantes selon leurs contribution aux axes factoriels. La typologie a ensuite été obtenue par la méthode de la classification ascendante hiérarchique (méthode de Ward).

${ }^{16}$ Bassin de vie rural : bassin de vie de l'INSEE polarisé par une commune appartenant à l'espace à dominante rurale.
} 
Côtes-d'Armor, de la Manche ou encore de la Somme. C'est également le cas de quelques bassins de l'Yonne, de la Nièvre, de l'Allier, du Cher et de l'Indre. Enfin, on note que certains de ces bassins sont encore très agricoles. Ces bassins « retraites et agriculture » se situent au cœur de la Bretagne, aux frontières du Limousin, du Centre et de Poitou-Charentes ou encore dans la Nièvre et en Côte-d'Or. Le profil «bassin de tourisme » est quasiment absent dans cette moitié nord métropolitaine.

Au sud d'une ligne La Rochelle-Annecy, on peut distinguer les départements " extérieurs », littoraux ou frontaliers, plus touristiques, et des départements intérieurs plus agricoles. Les bassins caractérisés par les retraites et l'agriculture sont concentrés dans le Cantal, l'Aveyron, le Gers ainsi qu'en Lozère et en Corrèze. Les bassins « retraites et tourisme » se situent soit plus à l'ouest jusqu'aux Pyrénées (Charente-Maritime, Dordogne, Lot, Gers, PyrénéesAtlantiques et Hautes-Pyrénées), soit dans les arrière-pays du pourtour méditerranéen et en Corse. Les bassins « retraites, tourisme et minima sociaux » sont typiques des départements méditerranéens, jusqu'au sud de l'Ardèche et à l'est de l'Ariège. Ce type de bassin apparaît aussi sur le littoral de la Gironde ou des Landes. Les bassins de tourisme décrivent les bassins ruraux alpins, pyrénéens, ou bien encore l'arrière-pays varois et certaines stations balnéaires de l'Atlantique.

Enfin, au sein de cette France rurale du sud, résidentielle et récréative, se distinguent quelques bassins plus productifs. Ils se situent dans la vallée du Rhône et aux alentours de Lyon, dans la périphérie éloignée de Toulouse, au contact des aires urbaines de Nîmes et Montpellier, ou encore de façon plus éparpillée dans l'extrême Sud-Ouest de la France.

Cette géographie souligne la diversité des dynamiques rurales. Notons que la diagonale aride $^{17}$, trop souvent présentée comme homogène, diffère d'une extrémité à l'autre. Le point commun à ces espaces pourrait être la présence relative d'une agriculture rurale couplée à d'autres activités productives au nord-est, à l'apport des retraités et du tourisme dans sa partie sud-ouest.

Revenus basiques différenciés, dynamiques de développement plus ou moins marquées... Une question émerge. Existerait-t-il des appariements de revenus basiques créant plus de développement que d'autres et qui expliqueraient la revitalisation de certains espaces ruraux ?

\section{Identifier les moteurs de la revitalisation rurale}

La synthèse des principales tendances démographique et socioéconomique pour chaque type de bassin rural fait émerger leurs spécificités.

\footnotetext{
${ }^{17}$ Cette expression est apparue dans les années 1980 à la DATAR pour désigner les territoires situés dans une diagonale allant des Ardennes à l'Ariège, marqués par le déclin des activités traditionnelles (agriculture et industrie) et subissant une forte érosion démographique.
} 


\section{$\underline{\text { Le rural productif }}$}

Il faut distinguer ici les territoires productifs périurbains («productif, migrant-alternant et Assedic » des territoires productifs et agricoles.

Les premiers regroupent $36 \%$ de la population rurale étudiée et jouxtent les aires urbaines. Les revenus du travail sont supérieurs au revenu moyen des bassins ruraux, mais les retraites restent faibles. Globalement, la progression des revenus des habitants y est moindre que dans les autres types de bassins ruraux sur la période 1990/2004. L'emploi salarié privé progresse également moins vite en raison d'une forte diminution des emplois dans les secteurs productifs et d'une plus faible progression des emplois dans les commerces et services de proximité. Cumulant des soldes migratoires et naturels positifs, la population, en revanche, est en forte croissance. On note, cependant, de forts taux d'émigration. Ces flux importants de sortants se conjuguent avec des prix immobiliers et fonciers relativement élevés sur le pourtour francilien.

La population croît sans qu'un impact positif sur les emplois, et notamment sur l'emploi domestique ne s'y fasse sentir. Les évasions de consommation, qui profiteraient aux pôles urbains proches, peuvent expliquer ces résultats. Cette déconnexion entre la démographique et l'emploi ou le revenu illustre le syndrome de la cité dortoir et les limites du modèle périurbain pour les espaces ruraux. Ainsi, le rural ne se développe pas forcément mieux à l'ombre des villes, si tant est que les marqueurs du développement soient l'emploi, les revenus, le chômage, etc. autant que la population.

Les bassins productifs et agricoles regroupent $23 \%$ de la population étudiée et apparaissent comme les plus productifs. La faiblesse des revenus par habitant est en partie liée à la faiblesse des niveaux des retraites et non des salaires. On note cependant une amélioration des retraites depuis 1999. L'emploi salarié y est plus dynamique que dans les espaces précédents, notamment grâce à la moindre diminution des secteurs productifs (industries agroalimentaires, mais aussi industrie mécanique, du métal ou de biens intermédiaires). Le faible coût du foncier peut constituer un avantage comparatif pour ces bassins, avantage qui s'est peut-être amenuisé dans les bassins précédents sous l'effet de la pression résidentielle. On note un meilleur taux d'équipements en commerces et services de proximité que dans les bassins précédents. Le maintien de la main-d'œuvre dans des activités locales semble limiter les évasions de consommation. En revanche, la population diminue en raison de faibles soldes migratoires, devenus positifs entre 1990 et 1999, qui ne compensent pas les soldes naturels, devenus négatifs entre 1990 et 1999. L'attractivité auprès des retraités peut, en partie, expliquer ce double renversement.

La situation de ces bassins est assez paradoxale. Peu attractifs, perdant de la population, encore fortement agricoles, ils n'en demeurent pas moins plutôt dynamiques en termes d'emplois et plutôt mieux équipés que les bassins précédents, plus «périurbains ». Spécialisés 
dans les activités de production «génériques ${ }^{18}$ soumises à la concurrence mondiale, leur équilibre peut paraître néanmoins fragile.

\section{$\underline{\text { Le rural des retraités }}$}

On a regroupé ici les bassins «retraites et agriculture» et «retraites et tourisme» qui représentent à eux deux $30 \%$ de la population étudiée. La fonction plus spécifiquement touristique des seconds se conjugue avec de meilleurs taux d'équipement en commerces et services aux particuliers. Les premiers, «retraites et agriculture », semblent plus pénalisés socialement. Le poids dans les revenus basiques du minimum vieillesse, des aides des départements aux personnes âgées, des allocations pour les handicapés y est plus important. Ce sont d'ailleurs ces bassins qui présentent les niveaux de revenus les plus faibles, même si l'écart à la moyenne se réduit après la prise en compte des revenus de transferts et du coût du sol. Cet écart à la moyenne se réduit également dans le temps, puisque ces bassins présentent les plus fortes progressions des revenus par habitant, en particulier des retraites. Le dynamisme de l'emploi salarié s'explique par l'essor des emplois domestiques associé, par endroits, à un dynamisme des secteurs productifs. L'analyse sectorielle souligne la présence d'activités productives spécifiques basées sur une ressource territorialement ancrée, activités d'autant plus dynamiques que le territoire est touristique.

Le développement de ces bassins paraît, certes, encore fragile ; mais le tourisme, l'arrivée de nouveaux retraités et la présence d'activités valorisantes et garantes des savoir-faire locaux en font des espaces ruraux moins pénalisés que ne le laisserait penser la seule variable population, en forte diminution.

\section{Le rural touristique}

Nous regroupons ici les bassins «retraites, tourisme et revenus sociaux » et les bassins de «tourisme », qui représentent $12 \%$ de la population rurale. A présent, les revenus basiques sont nettement dominés par les dépenses touristiques. Dans le premier type de bassin, caractéristique de l'arrière-pays languedocien, on note le poids considérable des revenus sociaux. Malgré tout, le revenu par habitant se situe à la moyenne des bassins de vie ruraux, cachant néanmoins de fortes disparités. Ces disparités entre niveaux de retraites (élevés) et revenus du travail (faible) sont encore plus visibles dans les bassins de tourisme. Elles laissent apercevoir les limites d'un modèle résidentiel qui entretiendrait ces inégalités, tout en étant un moyen efficace de lutter contre la pauvreté.

Dans ces deux types de bassins, les soldes migratoires sont positifs et compensent largement les pertes dues au solde naturel, qui ont tendance à s'amenuiser ; la population augmente rapidement. En revanche, dans les bassins les plus touristiques, on note de forts taux d'émigration. Ces bassins attirent de nouvelles populations mais en font également fuir. Fautil y voir un rapport avec le coût du foncier supporté par des salariés ou des indépendants pas nécessairement mieux rémunérés qu'ailleurs?

\footnotetext{
${ }^{18}$ Ces activités ne reposent pas sur les spécificités territoriales des zones d'implantation (comme un savoir-faire local ou un environnement particulier) mais sur des avantages comparatifs génériques (coût du foncier notamment).
} 
A ces mouvements migratoires résidentiels s'ajoute une forte présence touristique. Ainsi, la population présente à l'année est bien plus importante que la population effectivement recensée qu'elle dépasse de $35 \%$.

Les emplois progressent très rapidement, en raison d'une forte croissance des emplois domestiques déjà largement surreprésentés par rapport aux autres bassins ruraux, mais également des emplois dans le BTP. Les besoins en services à la personne qui accompagnent les mouvements migratoires résidentiels et touristiques semblent être d'importants pourvoyeurs d'emplois pour une population souvent peu qualifiée, mais peut-être faiblement rémunérée. Les conflits d'usage en la matière sont soulevés par les experts de ces questions (Perrier-Cornet, 2001 ; voir aussi les travaux du groupe Manon pour le Commissariat au plan).

La dynamique cumulée des revenus, des emplois et de la population semble donc directement liée aux ressources résidentielles de ces bassins, avec néanmoins quelques ombres au tableau en termes d'inégalités, de la faiblesse des revenus déclarés, ou encore l'apparition de taux d'émigration élevés dans les espaces les plus touristiques.

À la lecture de ce panorama rapide et synthétique, il semble que l'économie résidentielle joue un rôle décisif dans la revitalisation rurale. Plus précisément, c'est l'attractivité auprès des retraités et des touristes qui semble porteuse du dynamisme de l'emploi local et des revenus. En effet, les bassins résidentiels mais à caractère plus périurbain semblent souffrir du syndrome de la cité dortoir. La population croît rapidement, mais le développement escompté est limité par les évasions de consommation. Retenons également qu'une large partie des espaces ruraux sont encore fortement marqués par les activités de production « génériques ». L'emploi industriel y est, certes, en moindre recul qu'en moyenne en France, mais ces bassins semblent tout de même en délicate posture. Enfin, les territoires misant sur une production peut-être plus spécifique, assise sur des produits territorialement «marketés », semblent « s'en sortir » d'autant mieux qu'ils bénéficient des effets de levier du tourisme.

\section{Conclusion}

Une autre géographie des espaces ruraux que celle habituellement admise s'est dessinée. Son analyse montre que les tenants du développement local ne sont plus nécessairement ceux que l'on croyait, là où l'on pensait ; que les territoires non métropolitains profitent pleinement des mécanismes de redistribution privée et publique ; que des questions majeures se posent quant aux conséquences économiques et sociales, à l'équilibre et la durabilité de ces processus de développement local. Si les territoires touristiques et plus généralement résidentiels apparaissent plus dynamiques, ils révèlent également des aspects négatifs en termes d'inégalités et de taux d'émigration qu'il convient de souligner. Inversement, les bassins ruraux productifs semblent moins performants. Mais ils ne sont pas entièrement homogènes et apparaissent d'autre part non dépourvus d'atouts. Entre ces deux extrêmes, les bassins plus agricoles et moins touristiques semblent fragiles... mais les emplois y ont pourtant progressé plus rapidement que dans les bassins qui bénéficient du desserrement urbain et périurbain.

\footnotetext{
${ }^{19}$ Calcul de l'auteur d'après les données départementales de présence du ministère du Tourisme. On consultera à ce sujet le site de Christophe Terrier : http://www.christophe-terrier.com/
} 
Comme le soulignait déjà Perrier-Cornet (2004), le «tout résidentiel», comme le tout productif, ne paraît ni souhaitable, ni réaliste. «L'usage de nature » pour reprendre les termes de Perrier-Cornet, les politiques environnementales qui l'accompagnent ou encore la hausse des coûts de transport seront probablement des freins à ces dynamiques extrêmes. L'enjeu sera alors de concilier ces nouvelles nécessités et ces nouvelles contraintes avec le développement, récent et encore fragile, des espaces ruraux. 


\section{Bibliographie}

\section{Bibliographie}

AUBERT Francis, BLANC Michel (2002). Activités économiques et emplois : le rural refuge de secteurs déclassés ou milieu attractif? in Repenser les campagnes, (dir.) Philippe Perrier-Cornet, 2002, (coll.) bibliothèques des territoires, éditions de l'Aube, pp. 147-172.

BECATTINI Giacomo (1992). Le district marshallien : une notion socio-économique. In G. Benko et A. Lipietz (dir.), Les régions qui gagnent. Districts et réseaux : les nouveaux paradigmes de la géographie économique. Paris, PUF, pp. 35-55.

BONTRON Jean-Claude (1993). La reprise démographique confirmée. In Bernard Kayser (dir.), Naissances de nouvelles campagnes, DATAR, éditions de l'Aube, 1993, pp. 23-36.

CALLOIS Jean-Marc (2004). Can Quality Labels trigger rural Development? A microeconomic Model with Cooperation for the Production of a differentiated agricultural Good. Working Paper 2004/6, CESAER.

CANTILLON Richard (1952) Essai sur la nature du commerce en général. Paris, INED (première publication sans nom d'auteur en 1755 à Londres, chez Fletcher Gyles dans Holborn).

CERAMAC (2007). Habiter et vivre dans les campagnes de faible densité, acte du [2e] colloque franco-espagnol de géographie rurale, Foix, 15-16 septembre 2004 / ouvrage coordonné par Laurence Barthe [et al.], Ed. Clermont-Ferrand : Presses universitaires Blaise Pascal, 2007 ; Coll. CERAMAC, 785 p.

COISSARD Steven, PECQUEUR Bernard (2007). Des avantages comparatifs aux avantages différenciatifs : une approche par le territoire. Communication au XLIII ${ }^{\mathrm{e}}$ colloque de l'ASRDLF, Grenoble et Chambéry, 11-13 juillet 2007.

COLLETIS Gabriel, RYCHEN Frédéric (2004). Entreprises et territoires : proximité et développement local. In. (dir.) Pecqueur B., Zimmermann (J.B.), Économie et proximités, Lavoisier, coll. Hermès, 2004, pp. 207-228.

COMBES Pierre-Philippe, MAYER Thierry, THISSE Jacques-François (2006). Economie géographique. Ed. Economica, Coll. Corpus Economie, 400p.

COURLET Claude, PECQUEUR Bernard (1992). Les systèmes industriels localisés en France : un nouveau modèle de développement. In (dir.) Benko G., Lipietz A., Les régions qui gagnent, PUF, pp. 81-102.

DAVEZIES Laurent (2003). La diversité du développement local dans les villes françaises. Rapport à la DATAR. OEIL-CRETEIL, Université Paris-XII, Créteil, polyg. 60 p. (+ annexes).

DAVEZIES Laurent (2008). La République et ses territoires. La circulation invisible des richesses. Paris, Seuil, col. La République des idées, 2008, 110 p.

FRANçoIS Hugues, HIRCZACK Maud, SENIL Nicolas (2006). Territoire et patrimoine : la co-construction d'une dynamique et de ses ressources, Revue d'économie régionale et urbaine, 2006, vol. 5, pp. 683-700.

GAIGNE Carl (2000). Appariement et stabilité de la relation d'emploi dans les espaces ruraux. Revue d'économie régionale et urbaine, 2000, vol. 5, pp. 821-840.

GAIGNE Carl, GOFETTE-NAGOT Florence (2003). Localisation des activités en zones rurales : que nous enseigne l'économie géographique ? Working Paper GATE, 2003-03.

GONNARD Sophie (2001). Le procès de la base économique. Enquête sur l'interprétation, l'usage et la condamnation de la base économique par l'économie urbaine du XXe siècle. Mémoire de DEA, sous la direction de L. Davezies, Institut d'Urbanisme de Paris, Université Paris-XII, polyg. 58 p.

HOYT Homer (1954). Homer Hoyt on Development of Economic Base Concept. Land Economics, vol. 30 , pp182-186.

INSEE, INRA, IFEN, SCEES (2003). Structuration de l'espace rural: une approche par les bassins de vie. INSEE, Paris, juillet 2003, 56 p.

KAYSER (B.), (1990), La renaissance rurale, sociologie des campagnes du monde occidental, Paris : A. Colin.

KRUGMAN Paul (1991). Increasing Returns and Economic Geography. Journal of Political Economy 99, pp. 483-499. 
LAURENT Loeiz (1995). Le fonctionnement économique des bassins d'emploi. Réhabilitation de la théorie de la base. Division Statistiques et Études régionales de l'INSEE. Note d'Étude régionale $\quad \mathrm{n}^{\circ} \mathrm{H} 9506$. Paris, octobre 1995, 22 p.

MERLIN Pierre (1971). L’exode rural. (Coll.) Travaux et documents, PUF, n59, 350 p.

NORTH Douglas (1955). Location Theory and Regional Economic Growth. Journal of Political Economy, vol. 63, pp243-258.

PECQUEUR Bernard (1989). Le développement local. Éditions La Découverte et Syros, Paris, 2 e éd. 2000,132 p.

PECQUEUR Bernard, ZIMMERMANN Jean-Benoît (dir.) (2004) Économie de proximités. Lavoisier, coll. Hermès Science, Paris, 2004, 259 p.

PERRIER-CORNET (P.), (dir.), (2002), Repenser les campagnes, La Tour d'Aigues : Editions de l'Aube.

PERRIER-CORNET Philippe (2004). L'avenir des espaces ruraux français, dynamiques et prospective à l'horizon 2020. Futuribles, 299, p.77-95.

RENARD Jean (2000). Conclusion du colloque européen consacré aux campagnes vivantes tenu à Nantes les 18 et 19 mai 2000, dont les actes ont été publiés : Nicole CROIX [dir.], Des campagnes vivantes, un modèle pour l'Europe ? Mélanges offerts au professeur Jean Renard, Rennes, Presses universitaires de Rennes, 2000, 696 p.

SENCEBE Yannick, LE PICIER Denis (2005). Migrations résidentielles de l'urbain vers le rural : différenciation sociale des profils et ségrégation spatiale. Communication au colloque Mobilité, différenciations et inégalités, 21-22 avril 2005 - Lyon / Vaulx-en-Velin

SOMBART Werner (1916). Der moderne Kapitalismus. Munich, Duker et Humbolt. Vol. 3.

TALANDIER Magali (2007). Un autre modèle de développement hors mondialisation. Le cas du monde rural français. Thèse de doctorat, CRETEIL-Institut d'Urbanisme de Paris, Université Paris-XII, polyg. 379 p. + annexes ; disponible à l'adresse http://talandier.com/thesetalandier.pdf

TIEBOUT Charles (1956). Exports and Regional Economic Growth. Journal of Political Economy, vol.64, pp160-164. 